\title{
A Rap GTPase interactor, RADIL, mediates migration of neural crest precursors
}

Gromoslaw A. Smolen, ${ }^{1}$ Benjamin J. Schott, ${ }^{1}$ Rodney A. Stewart, ${ }^{2}$ Sven Diederichs, ${ }^{1}$ Beth Muir, ${ }^{3}$ Heather L. Provencher, ${ }^{3}$ A. Thomas Look, ${ }^{2}$ Dennis C. Sgroi, ${ }^{3}$ Randall T. Peterson, ${ }^{4}$ and Daniel A. Haber ${ }^{1,5}$

${ }^{1}$ Massachusetts General Hospital Cancer Center, Harvard Medical School, Charlestown, Massachusetts 02129, USA; ${ }^{2}$ Department of Pediatric Oncology, Dana-Farber Cancer Institute, Boston, Massachusetts 02115, USA; ${ }^{3}$ Department of Pathology, Massachusetts General Hospital, Molecular Pathology Research Unit, Harvard Medical School, Charlestown, Massachusetts 02129, USA; ${ }^{4}$ Cardiovascular Research Center, Massachusetts General Hospital, Harvard Medical School, Charlestown, Massachusetts 02129, USA

The neural crest (NC) is a highly motile cell population that gives rise to multiple tissue lineages during vertebrate embryogenesis. Here, we identify a novel effector of the small GTPase Rap, called RADIL, and show that it is required for cell adhesion and migration. Knockdown of radil in the zebrafish model results in multiple defects in NC-derived lineages such as cartilage, pigment cells, and enteric neurons. We specifically show that these defects are primarily due to the diminished migratory capacity of NC cells. The identification of RADIL as a regulator of NC migration defines a role for the Rap pathway in this process.

Supplemental material is available at http://www.genesdev.org.

Received April 12, 2007; revised version accepted July 13, 2007.

During vertebrate development, precursor cells of various lineages undergo a complex set of morphogenetic movements to assume their final position in the embryo. Implementation of such movements depends on both the patterns set up by several morphogens such as Wnts, retinoic acid, bone morphogenic proteins (BMPs), and fibroblast growth factors (FGFs), as well as the ability of cells to respond to these signals by executing cell motion. Regulation of cell adhesion has emerged as a common theme in a number of settings where cellular precursors traverse large distances to assume their final differentiated state, but the underlying molecular mechanisms are poorly defined.

The zebrafish model offers an ideal experimental system for the evaluation of cell adhesion and cell motility

[Keywords: Neural crest; migration; adhesion; Rap; development; zebrafish]

${ }^{5}$ Corresponding author.

E-MAIL haber@helix.mgh.harvard.edu; FAX (617) 724-6919.

Article published online ahead of print. Article and publication date are online at http://www.genesdev.org/cgi/doi/10.1101/gad.1561507. during embryogenesis. The neural crest $(\mathrm{NC})$ cells, a transient population of multipotent progenitors arising at the lateral edge of the neural plate in vertebrate embryos, migrate extensively to give rise to a number of cell types such as the craniofacial skeleton, neurons and glia of the peripheral nervous system, and pigment cells (Le Douarin and Kalcheim 1999|. Their migration requires active adhesion changes during both the epithelial-tomesenchymal transition at the dorsal neural tube and their subsequent navigation along stereotypical pathways to their final position. The importance of NC migration during normal development is highlighted by the human constitutional defects associated with NC function, which often result in craniofacial abnormalities and account for up to $30 \%$ of all congenital birth defects (Jones and Trainor 2004). In some cases, specific genetic abnormalities have been identified, as in mutations in the adhesion/migration factor Ephrin B1 (EFNB1) in craniofrontonasal syndrome (Wieland et al. 2004), deletions of the migration-inducing gene SLUG in Waardenburg syndrome (WS2D) (Sanchez-Martin et al. 2002), and mutations in GDNF and its receptor RET, two important regulators of NC migration, in Hirschsprung disease (Edery et al. 1994; Ivanchuk et al. 1996; Iwashita et al. 2003). However, the majority of NC defects are unexplained at the molecular level.

The low-molecular-weight GTPase Rap is a member of the Ras GTPase superfamily encoding molecular switches that cycle between inactive GDP-bound and active GTP-bound states. Rap appears to have an evolutionarily conserved role in regulating adhesion and migration in a wide range of species, ranging from Dictyostelium to vertebrates (Bos et al. 2001; Caron 2003). However, defining the function of Rap in early vertebrate embryonic development is complicated by the presence of multiple Rap genes, and thus analysis of unique Rap interactors using a model organism may provide valuable information about this complex pathway.

In this study, we identify a novel downstream effector of Rap, RADIL (RA [Ras association] and DIL domains), which plays a critical role in cell adhesion and migration. Loss of Radil function in zebrafish embryos results in defects in NC migration. These observations point to a critical role for the Rap pathway in developmentally regulated cellular migration of NC and, more broadly, the role of cell adhesion in morphogenetic movements shaping the vertebrate embryo.

\section{Results and Discussion}

\section{Interactions of RADIL with Rap}

We initially identified RADIL in a screen for genes induced by an oncogenic translocation in a pediatric sarcoma (data not shown). While its role in cellular transformation appears to be modest, its domain architecture and effects on cellular migration prompted us to pursue its role in normal development. Human RADIL encodes a novel protein of 1075 amino acids, including RA, DIL, and PDZ domains (Supplementary Fig. S1). The RA domain is present in a number of downstream effectors of Ras and Rap, and interacts in vivo with these two GTPases, displaying varying degrees of specificity between them. The function of the DIL domain is un- 
known, but it is found in several cytoskeleton-associated proteins, such as myosin V. Finally, the PDZ domain at the $\mathrm{C}$ terminus represents a well-known protein-protein interaction platform. The domain architecture of RADIL is most closely related to AF6, an important regulator of cell adhesion that serves as an intermediary between membrane-bound proteins and actin cytoskeleton. In fact, RADIL has been described as AF6-like in a recent report characterizing the role of AF6 in cell adhesion (Zhang et al. 2005). However, in the absence of significant functional similarity between the two proteins, we propose the name RADIL, reflecting its domain architecture.

To investigate fully the GTPase-binding preference of RADIL, we first cotransfected a Flag-tagged RADIL expression construct along with HA-tagged GTPases into AD293 cells. Immunoprecipitation using anti-Flag antibody, followed by immunoblotting with anti-HA antibody, clearly shows that RADIL preferentially binds to Rap, but not to any of the Ras family proteins (Fig. 1A). To test whether RADIL binding is dependent on the GTPase activation status, we cotransfected RADIL with constitutively active Rap (Rap E63) and dominant-negative (Rap N17) mutants (Fig. 1B). RADIL was effectively coimmunoprecipitated only with the constitutively active Rap mutant, suggesting that this interaction is dependent on the Rap-GTP state. Further demonstrating specificity, no interaction was observed with two additional GTPases-cdc42 and Rac (data not shown).
To investigate the role of RADIL in a mammalian tissue culture system, we knocked down endogenous Radil expression using lentiviral short hairpin RNAs (shRNAs) in a mouse breast epithelial cell line NMuMG. Given the well-described role of Rap and its effectors in cell adhesion, we performed short-term adhesion assays. Two independent hairpins achieved partial knockdown of Radil expression to $\sim 40 \%$ of control cells, as determined by quantitative TaqMan PCR (qPCR) (Fig. 1D). Radil knockdown resulted in dramatic reduction of the adhesive properties of these cells to $<10 \%$ of control cells (Fig. 1C), suggesting that RADIL is involved in modulating cellular adhesion.

To assess the role of RADIL in cellular migration, we also knocked down RADIL in a highly migratory human chondrosarcoma cell line, SW1353. Reduced RADIL expression $(\sim 50 \%)$ using two independent small interfering RNA (siRNA) duplexes (Fig. 1F), resulted in decreased (to $\sim 40 \%$ ) migratory capacity of these cells (Fig. 1E). RADIL therefore contributes to both cell adhesion and migration, properties that have been linked to Rap signaling. Ectopic overexpression of RADIL did not further enhance migration or adhesion of the parental cells used in either of these assays (data not shown), indicating that RADIL may be required, but is not the limiting component of these processes.

Recent evidence has suggested that some Rap effector molecules can interact with Rap GAPs, thus regulating the levels of active Rap-GTP through a direct feedback
A

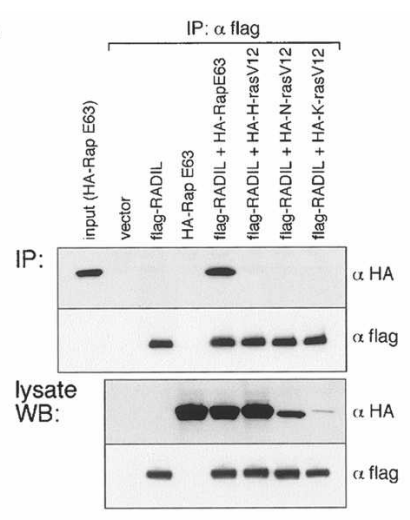

B

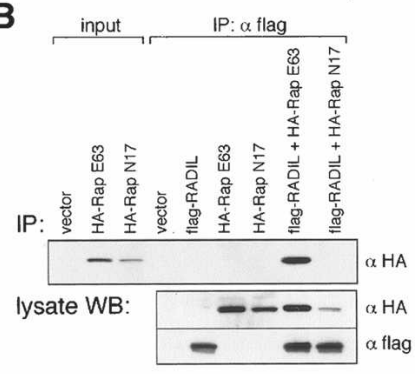

C

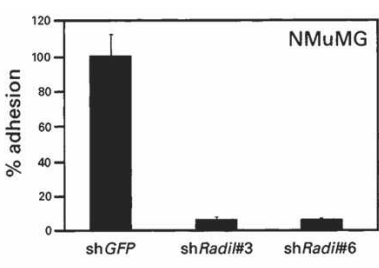

E

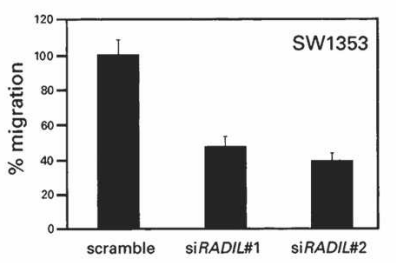

G

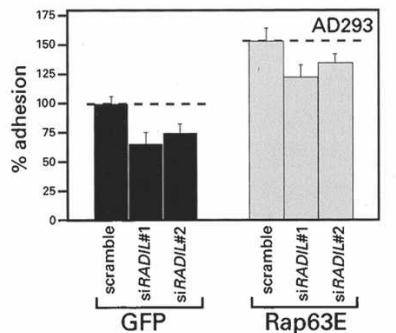

D

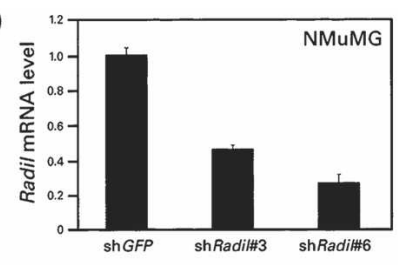

$\mathbf{F}$

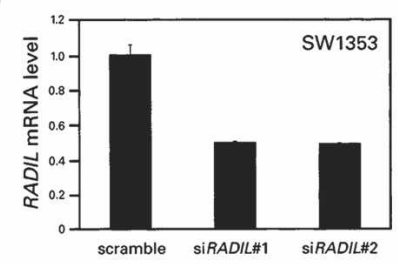

H

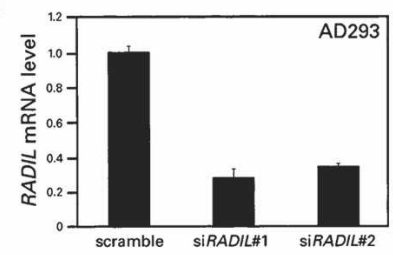

Figure 1. RADIL binds to activated Rap GTPase and is involved in cell adhesion and migration. (A) Immunoprecipitation of Flag-tagged RADIL with HA-tagged GTPases, showing preferential association of RADIL with Rap and not with H-ras, N-ras, or K-ras. $(B)$ Immunoprecipitation of Flag-tagged RADIL with HA-tagged constitutively active Rap (RapE63), but not with dominant-negative (RapN17). (C) NMuMG cells with decreased Radil mRNA levels display defects in adhesion. $(D)$ qPCR showing relative Radil mRNA levels. Radil knockdown was achieved in mouse NMuMG cells using two independent lentiviral hairpin constructs. (E) SW1353 cells with decreased RADIL levels display defects in migration. (F) qPCR showing relative RADIL mRNA levels. RADIL knockdown was achieved in human SW1353 cells using two independent siRNA duplexes. $(G)$ RADIL is downstream from Rap. Adhesion defects in AD293 cells upon knockdown of endogenous RADIL expression cannot be fully suppressed by overexpression of constitutively active Rap63E, suggesting that RADIL is a downstream effector of Rap. (H) qPCR showing relative RADIL mRNA levels. RADIL knockdown was achieved in human AD293 cells using two independent siRNA duplexes. 
loop (Su et al. 2003). Elucidation of epistatic relationships is therefore critical in ordering the pathway. We tested the effect of either overexpression of constitutively active RapE63 or knockdown of endogenous RADIL in the highly transfectable cell line AD293. Knockdown of RADIL (to $\sim 30 \%$ ) was achieved using two independent duplexes (Fig. $1 \mathrm{H}$ ) and resulted in a significant decrease in cellular adhesion to $\sim 70 \%$ of control cells (Fig. 1G). While expression of constitutively active Rap resulted in increased adhesion at baseline in this cell line, it did not abrogate the effect of RADIL knockdown, suggesting that RADIL may function downstream from Rap. As such, RADIL would be predicted not to affect the levels of the Rap-GTP active state. Indeed, overexpression of RADIL in AD293 cells did not affect the relative levels of Rap-GTP (data not shown). Together with the physical interaction between RADIL and the active GTP-bound Rap, these results suggest that RADIL may be a downstream effector of Rap.

RA domain proteins have been shown to bind to Ras GTPase and may display some promiscuity in their GTPase selection, particularly under in vitro conditions. To confirm that RADIL does not impinge on Ras-dependent signaling, we used two standard readouts for Ras function: focus formation in 3T3 cells and a Ras-dependent transactivation assay. Whereas titration of Rasbinding proteins can result in modulation of Ras-dependent output (Peterson et al. 1996; Shao and Andres 2000), increasing concentrations of RADIL did not affect the number of foci formed in 3T3 cells (data not shown). A standard Ras-responsive promoter was also unaffected by RADIL overexpression, either at basal or Ras-activated levels of reporter readout (Supplementary Fig. S2). Thus, RADIL appears to interact specifically with active Rap and does not modulate Ras signaling.

\section{Disruption of Radil function in zebrafish}

To study the in vivo function of RADIL in vertebrate development we cloned a full-length zebrafish cDNA. Zebrafish radil encodes a protein of 1125 amino acids with $49 \%$ identity and $64 \%$ similarity to human RADIL. The level of radil expression in zebrafish is regulated over the course of $7 \mathrm{~d}$ of development (Fig. 2A). Very low but detectable levels of radil transcript are present in one-cell embryos, suggesting the presence of maternally deposited transcripts and the levels of radil increase upon the onset of zygotic transcription (around the 1000cell stage), eventually peaking at $72 \mathrm{~h}$ post-fertilization (hpf). To investigate the spatial distribution of radil transcripts, we performed in situ hydridization using radilspecific probe (Supplementary Fig. S3). The expression pattern appeared to be ubiquitous, and enriched in the anterior part of the embryos.

To investigate the consequences of abrogation of radil function in zebrafish, we used an antisense morpholino (MO) knockdown approach. A number of specific defects affecting craniofacial cartilage, enteric neurons, cranial ganglia, glial cells of the lateral line, as well as iridophore and xanthophore pigment cells were evident (Fig. 2C-H; Supplementary Fig. S4). Other lineages, such as melanophores and dorsal root ganglia, appeared to be unaffected or only marginally affected (Supplementary Fig. S4). These effects were specific to radil gene knockdown as judged by several criteria. Three independent MOs /one targeting the translational start, ATG MO, and the other
A
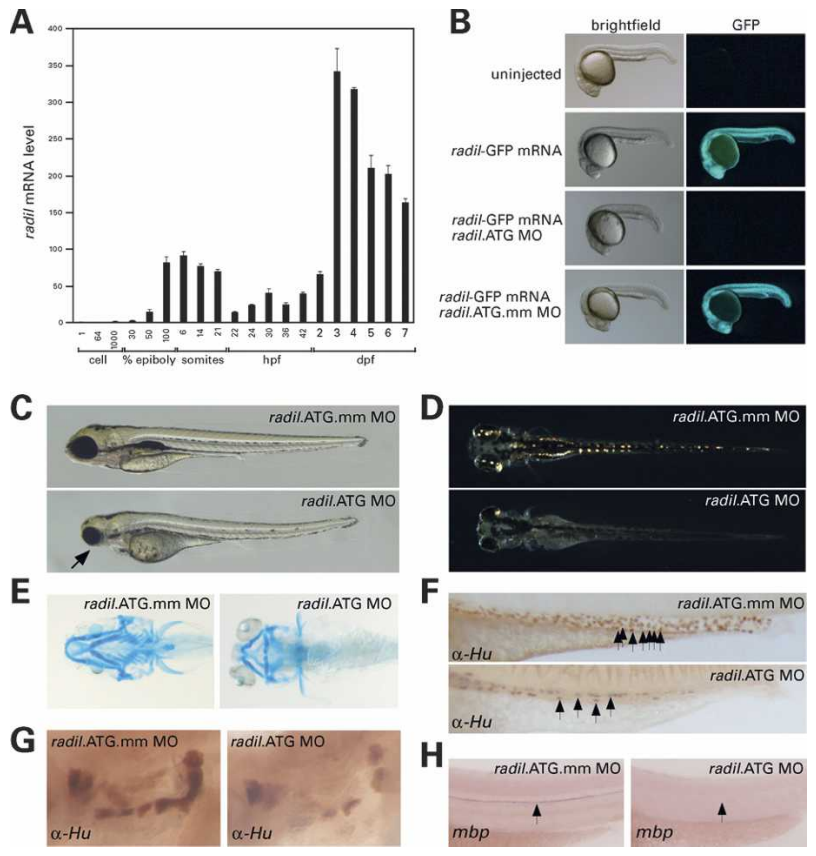

Figure 2. Characterization of radil knockdown phenotypes. $(A)$ qPCR showing relative radil mRNA levels during early zebrafish embryogenesis. (B) Specificity of radil.ATG MO. Translational fusion of the first 25 nucleotides of radil ORF to GFP is efficiently suppressed by the ATG MO, but not by the mismatch control MO. Embryos at $24 \mathrm{hpf}$ are shown. (C) Morphology of radil knockdown embryos at $4 \mathrm{dpf}$, compared with the mismatch control-injected embryos. Arrow points to the jaw defect. $(D)$ Iridophore pigmentation defects in radil knockdown embryos at $4 \mathrm{dpf}$ compared with the mismatch control-injected embryos. (E) Craniofacial defects in radil knockdown embryos at $7 \mathrm{dpf}$ compared with the mismatch controlinjected embryos. Cartilage was stained with Alcian Blue. $(F)$ Enteric neuron defects in radil knockdown embryos at $4 \mathrm{dpf}$ compared with the mismatch control-injected embryos, visualized with anti-Hu antibody staining. Arrows point to individual enteric neurons, highlighting the decreased numbers in radil morphants. $(G)$ Cranial ganglia defects in radil knockdown embryos at 4 dpf compared with the mismatch control-injected embryos, visualized with anti-Hu antibody staining. $(H)$ Significant reduction in the glial cells of the lateral line in radil knockdown embryos at 3 dpf compared with the mismatch control-injected embryos, visualized by in situ hybridization with $m b p$ (myelin basic protein) probe.

two targeting splice donor sites of exons 4 and 14) resulted in similar phenotypes (Supplementary Table S1). The exon 4 donor splice site MO resulted in the use of a cryptic splice donor site within exon 4 and is predicted to produce an in-frame deletion of 57 amino acids within the Radil protein (Supplementary Fig. S5). The exon 14 donor splice site MO resulted in the incorporation of an intron and premature Radil protein truncation (Supplementary Fig. S6). No phenotypes were observed upon injection of three corresponding mismatch MOs at the same concentrations. Furthermore, injection of increasing concentrations of radil.ATG MO resulted in progressively more severe phenotypes demonstrating a doseresponse relationship (Supplementary Fig. S7). Consistent with the specificity of these MOs, translational fusion of the first 25 nucleotides of radil to GFP was efficiently suppressed by the ATG MO but not by the mismatch control MO (Fig. 2B).

The cell types affected by radil knockdown point to a defect with early NC development. The initial induction 
A
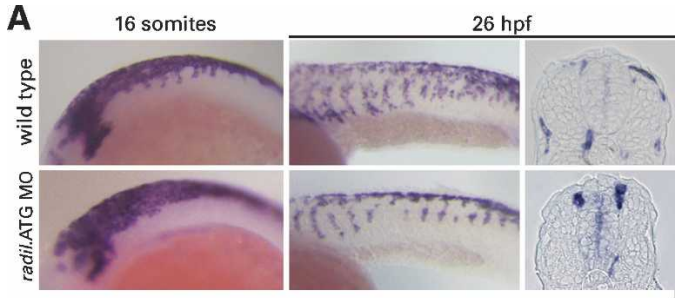

B

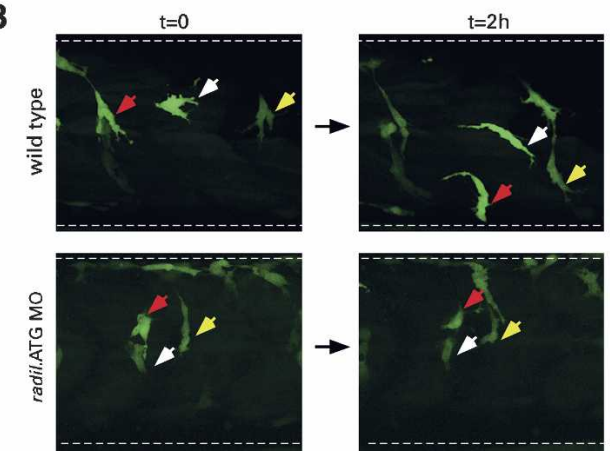

Figure 3. radil knockdown results in defects of NC migration. $(A)$ Defect in early NC migration in radil knockdown embryos, as visualized by in situ hybridization with crestin marker. Embryos were fixed at the 16-somite stage and $26 \mathrm{hpf}$. Sections were taken at the 26-hpf time point, halfway through the yolk extension. $(B)$ Defect in early NC migration in radil knockdown embryos, as visualized using the foxD3:GFP transgenic line. Panels shown represent frames taken from Supplementary Movies 1 and 2. Movies were started at $\sim 24 \mathrm{hpf}$ and 31 hpf for wild-type and radil mophants, respectively. The dotted white lines mark the top of the embryo and the edge of the yolk extension. Arrows (white, yellow, and red) identify individual cells and their respective changes in position over time.

of NC is not affected in the radil morphant embryos at the six-somite stage (Supplementary Fig. S8), as indicated by robust expression of two early NC markers foxd3 and sox10. Subsequent migration of these cells, however, showed profound defects. We used the pan-NC marker crestin to follow cell migration (Fig. 3A; Supplementary Fig. S12). At the 16-somite stage, wild-type embryos clearly show migrating NC cells, but radil morphant embryos have significant impairment with no NC cell migration evident at this stage. At the 26-hpf stage, crestin-positive cells in wild-type embryos have reached the yolk extension, whereas radil morphants have fewer crestin-positive cells, some of which have not migrated as far, while others have remained in their original positions at the neural keel. To demonstrate the migration defects at single-cell resolution, we took advantage of the (foxD3:GFP) transgenic line, in which NC cells are tagged and can be monitored by real-time imaging (Gilmour et al. 2002). Knockdown of radil resulted in defects in gross cell migration compared with wild-type embryos (Fig. 3B; Supplementary Movies 1, 2).

To further characterize the NC defects, we undertook a more detailed analysis of craniofacial development (Supplementary Fig. S9). Expression of $d 1 \times 2 a$, which at $24 \mathrm{hpf}$ is expressed in migratory NC that forms pharyngeal arches, was mildly affected in radil morphants, particularly in the hyoid and branchial arches. However, dlx2a expression by $48 \mathrm{hpf}$ clearly showed significant reduction of mandibular and hyoid arches and absence of branchial arches in radil morphants. Similarly, the staining pattern of gsc, a marker of early chondrocytes, showed a dramatic reduction in radil morphants at 48 hpf. Together, these finding are consistent with a progressive defect in NC migration, resulting in insufficient numbers of migrating NC cells populating the pharyngeal pouches, which leads to loss of early chondrocyte markers and craniofacial cartilage defects observed at 7 dpf (Fig. 2).

The migration defect in NC precursors in radil morphants is associated with a reduced number of NC derivatives at later stages of development. To determine whether these cells have an increased rate of apoptosis, we measured cell death in radil morphants using TUNEL staining. Knockdown of radil was associated with a dramatic increase in apoptosis compared with the uninjected controls, as well as the mismatch control MO-injected embryos (Fig. 4; Supplementary Fig. S10). To distinguish between NC cell apoptosis and failure of migration as the primary cause of developmental defects in radil morphants, we suppressed cell death using overexpression of the zebrafish bcl2 anti-apoptotic gene (Liu et al. 2003; Langenau et al. 2005). Injection of the radil ATG MO together with bcl2 mRNA led to efficient rescue of apoptosis measured at $24 \mathrm{hpf}$ (Fig. 4), as well subsequent time points until $5 \mathrm{dpf}$ (Supplementary Fig. S11). However, these embryos showed no rescue of cartilage, enteric neurons, or iridophore defects (Fig. 4). Similar findings were observed when apoptosis was suppressed using functional inactivation of p53 by coninjecting $\mathrm{ra}$ dil.ATG MO with p53 MO (Robu et al. 2007; data not shown). Furthermore, bcl2 mRNA coninjection did not rescue the migration defect of crestin-positive cells at 24 hpf (Supplementary Fig. S12). Thus, the induction of apoptosis and migration defects can be uncoupled in radil morphant embryos, providing evidence that migration defects may be primarily responsible for the phenotypes observed in these embryos, which is consistent with our findings in mammalian cells showing that RADIL expression is required for normal cell adhesion and migration.

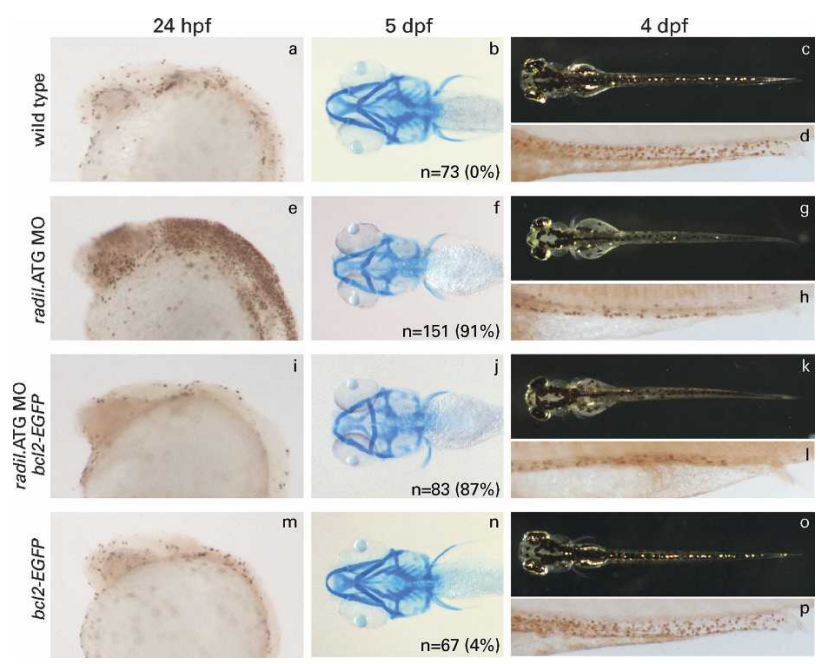

Figure 4. Apoptosis is not the primary cause of the abnormalities of NC-derived lineages in radil morphants. $(a, e, i, m)$ Knockdown of radil in zebrafish results in the onset of apoptosis, as shown by TUNEL assay. bcl2 overexpression can rescue apoptosis in radil morphants at $24 \mathrm{hpf}$, but does not rescue craniofacial $(b, f, i, n)$, iridophore $(c, g, k, o)$, or enteric neuron $(d, h, l, p)$ defects later in development. Sample size $(n)$ and the percentage of embryos with craniofacial abnormalities are indicated. 


\section{Concluding remarks}

In this study, we describe an effector of Rap, termed RADIL, which displays a preference for Rap-GTP binding and modulates Rap-dependent processes including cell adhesion and migration. To gain in vivo insight into the function of RADIL, we used the zebrafish model system and identified the highly motile NC lineage as being particularly affected by radil knockdown. Defects in such a diverse set of differentiated cells as cartilage, iridophores, and enteric neurons suggest an early defect at a level of undifferentiated precursor NC cells. Using a combination of approaches, we showed that impaired adhesive/migratory properties of NC cells may underlie the observed phenotypes.

Rap has been strongly implicated in the regulation of cellular adhesion in multiple biological settings (Bos 2005). However, given the multiplicity of Rap functions as well as genetic redundancy of the Rap family in most organisms, studies of individual Rap regulators and effectors have been most informative in elucidating specific Rap functions during development. While the mouse knockout of AF6 resulted in early embryonic lethality due to abnormal cell-cell junctions (Zhadanov et al. 1999), other Rap effector knockouts have shown very specific phenotypes: For instance, RapL knockout mice display defects only in adhesion and migration of lymphocytes and dendritic cells (Katagiri et al. 2004). Given that the unifying theme of Rap function studies appears to be the regulation of cadherin- and integrin-based adhesion, in vivo studies are critical in providing the relevant physiological context.

Modulation of adhesion has emerged as an important player in early morphogenetic cell movements (SolnicaKrezel 2006). In the first study to globally abrogate Rap function in zebrafish, defects in convergence and extension movements during gastrulation, which involve dorsoventral narrowing (convergence) and anterioposterior lengthening (extension) of embryonic tissues, were recently observed (Tsai et al. 2007). This strong effect on early embryo morphogenetic movements makes the assessment of later roles of Rap quite challenging. However, a role for Rap and adhesion modulation is likely in later developmental processes, such as the NC migration, as suggested by the significant defects observed in radil morphant embryos.

Modulation of cellular adhesion through integrins and cadherin has been shown to have a profound impact on NC migration. Conditional deletion of $\beta 1$ integrin by Ht-PA-Cre, expressed specifically in NC cells at the start of migration, leads to severe Hirschsprung-like phenotypes in mice (Breau et al. 2006). Cadherin-11 in mouse and Xenopus and Cadherin-7 in chick are specifically expressed only in the migratory NC and not in the earlier premigratory NC. However, forced overexpression of Cadherin-11 in Xenopus and Cadherin-7 in chick results in failure of NC migration (Nakagawa and Takeichi 1998; Borchers et al. 2001). Therefore, a complex picture emerges where NC migration is dependent on spatial, as well as temporal, regulation of NC cell adhesion. The identification of RADIL provides a link between NC migration and a well-known adhesion mediator, Rap, revealing another key component of this important biological pathway. Future identification of RADIL-interacting proteins is likely to elucidate a more complete molecular framework for RADIL function in regulating cell adhesion and migration.
Phenotypic comparison of radil morphants with known NC mutants could also yield insight into the pathways where Radil might be involved. During early neural development multipotent NC cells restrict their fate potential into ectomesenchymal and nonectomesenchymal pools (Kelsh and Eisen 2000). Ectomesenchymal NC derivatives include craniofacial skeleton and fin mesenchyme, while nonectomesenchymal NC derivatives include pigment cells, neurons, and glia. Analysis of phenotypes in zebrafish sox10 mutants has been clearly shown to affect only the nonectomesenchymal NC lineages (Kelsh and Eisen 2000; Dutton et al. 2001). In contrast, radil morphants display more broad defects, suggesting that both pools of NC precursors are affected. Zebrafish tfap2a mutants display phenotypes in both NC precursor pools, but the primary defect appears to be in early patterning of NC subpopulations (Knight et al. 2003; Barrallo-Gimeno et al. 2004). While NC migration appears to be a major defect in radil morphants, tfap $2 a$ mutants display modest if any aberrant cell migration. Consistent with the patterning defect in tfap2a mutants, embryos display absence of entire prechondrogenic NC streams that populate pharyngeal arches at 24 hpf. In contrast, expression of dlx $2 a$ in radil morphants is only modestly affected at $24 \mathrm{hpf}$, but dramatically reduced at $48 \mathrm{hpf}$, arguing for a later role of Radil compared with Tfap2a. Zebrafish foxd3 mutants exhibit a complex phenotype with defects in NC specification, survival, and migration (Lister et al. 2006; Stewart et al. 2006). Given the substantial phenotypic overlap with radil morphants and the involvement of Foxd3 in NC migration, we tested whether radil expression was affected foxd3 mutants. While no difference in radil expression was observed (data not shown), it remains possible that other Foxd3 target genes may functionally interact with Radil.

A common phenotype frequently observed in the NC mutants is the induction of apoptosis. Functional abrogation of several classes of molecules can lead to NC apoptosis, including adhesive molecules ( $\alpha 4 \beta 1$ integrin), signaling molecules (Fgf8, Fgfr1), and transcription factors (foxd3, tfap2a, and foxi) (Abu-Issa et al. 2002; Nissen et al. 2003; Trokovic et al. 2003; Barrallo-Gimeno et al. 2004; Stewart et al. 2006). Collectively, these studies suggest that disruption of NC specification, migration, survival, or differentiation, can lead to increased cell death. However, in most cases the causal relationship between apoptosis and any of the above steps is unclear. The analysis of radil knockdown is particularly interesting in this context, in that its effects appear linked to cell migration, as opposed to NC specification, and that suppression of apoptosis in radil morphants by overexpressing the anti-apoptotic gene $b c 12$ or knocking down p53 function fails to rescue the appropriate development of craniofacial cartilage or other NC-derived lineages. Thus, our data suggests that adhesion/migration defects underlie the phenotypes observed, and apoptosis may be a secondary result of failure of cells to establish their appropriate developmental context.

Taken together, genetic evidence strongly suggests that defects in NC migration can lead to abnormalities in NC-derived lineages. In this report we provide evidence for the role of the Rap pathway in NC migration, raising the possibility that Rap regulators and effectors may be candidates for genes involved in human neurocristopathies. 


\section{Materials and methods}

MOs

All MOs were ordered from GeneTools. MOs used were as follows: radil.ATG, GGCAGATGAGGAACCGTAGAACATG; radil.ATG.mm, GGCAcATcAGGAAgCGTAcAAgATG; radil.ex4d, ATTTCGGCTGA CCTGTTGGTTGTAG; radil.ex4d.mm, ATTTaGGCTcACCTcTTGa TTcTAG; radil.ex14d, CAGCACACACTCACAAGTCCATCGA; radil. ex14d.mm, CAcCAgACACTCAgAAGTCgATgGA; p53, GCGCCATT GCTTTGCAAGAATTG (Robu et al. 2007). Mismatches in the control MOs are indicated in lowercase.

MOs were dissolved in $1 \times$ Danieau's buffer and injected at indicated concentrations (Table 1) into the yolk of one-cell stage embryos in a 1-nL volume. All of the analyses presented were conducted with embryos injected with 4 or $5 \mathrm{ng}$ of radil.ATG MO.

bcl2 overexpression

blc2-EGFP mRNA was synthesized as described previously (Liu et al. 2003). Two-hundred picograms of mRNA were injected per embryo. For the $b c 12$ rescue of apoptosis experiment, radil.ATG MO was injected at 4 ng per embryo.

Further information of about materials and methods is provided in the Supplemental Material.

\section{Acknowledgments}

This work was supported by Doris Duke Foundation Distinguished Clinical Investigator Award, National Foundation for Cancer Research grant, and National Institutes of Health grant P01 95281 (to D.A.H); National Institutes of Health CA1 17737 (to G.A.S); National Institutes of Health HL079267 (to R.T.P.); National Institutes of Health CA104605 (to A.T.L.); and National Institutes of Health K99NS058608 and Claudia Adams Barr Foundation (to R.A.S).

\section{References}

Abu-Issa, R., Smyth, G., Smoak, I., Yamamura, K., and Meyers, E.N. 2002. Fgf8 is required for pharyngeal arch and cardiovascular development in the mouse. Development 129: 4613-4625.

Barrallo-Gimeno, A., Holzschuh, J., Driever, W., and Knapik, E.W. 2004. Neural crest survival and differentiation in zebrafish depends on mont blanc/tfap2a gene function. Development 131: 1463-1477.

Borchers, A., David, R., and Wedlich, D. 2001. Xenopus cadherin-11 restrains cranial neural crest migration and influences neural crest specification. Development 128: 3049-3060.

Bos, J.L. 2005. Linking Rap to cell adhesion. Curr. Opin. Cell Biol. 17: $123-128$.

Bos, J.L., de Rooij, J., and Reedquist, K.A. 2001. Rap1 signalling: Adhering to new models. Nat. Rev. Mol. Cell Biol. 2: 369-377.

Breau, M.A., Pietri, T., Eder, O., Blanche, M., Brakebusch, C., Fassler, R., Thiery, J.P., and Dufour, S. 2006. Lack of $\beta 1$ integrins in enteric neural crest cells leads to a Hirschsprung-like phenotype. Development 133: 1725-1734.

Caron, E. 2003. Cellular functions of the Rap1 GTP-binding protein: A pattern emerges. J. Cell Sci. 116: 435-440.

Dutton, K.A., Pauliny, A., Lopes, S.S., Elworthy, S., Carney, T.J., Rauch, J., Geisler, R., Haffter, P., and Kelsh, R.N. 2001. Zebrafish colourless encodes sox10 and specifies non-ectomesenchymal neural crest fates. Development 128: 4113-4125.

Edery, P., Lyonnet, S., Mulligan, L.M., Pelet, A., Dow, E., Abel, L., Holder, S., Nihoul-Fekete, C., Ponder, B.A., and Munnich, A. 1994. Mutations of the RET proto-oncogene in Hirschsprung's disease. $\mathrm{Na}$ ture 367: 378-380.

Gilmour, D.T., Maischein, H.M., and Nusslein-Volhard, C. 2002. Migration and function of a glial subtype in the vertebrate peripheral nervous system. Neuron 34: 577-588.

Ivanchuk, S.M., Myers, S.M., Eng, C., and Mulligan, L.M. 1996. De novo mutation of GDNF, ligand for the RET/GDNFR- $\alpha$ receptor complex in Hirschsprung disease. Hum. Mol. Genet. 5: 2023-2026.

Iwashita, T., Kruger, G.M., Pardal, R., Kiel, M.J., and Morrison, S.J. 2003. Hirschsprung disease is linked to defects in neural crest stem cell function. Science 301: 972-976.
Jones, N.C. and Trainor, P.A. 2004. The therapeutic potential of stem cells in the treatment of craniofacial abnormalities. Expert Opin. Biol. Ther. 4: 645-657.

Katagiri, K., Ohnishi, N., Kabashima, K., Iyoda, T., Takeda, N., Shinkai, Y., Inaba, K., and Kinashi, T. 2004. Crucial functions of the Rap1 effector molecule RAPL in lymphocyte and dendritic cell trafficking. Nat. Immunol. 5: 1045-1051.

Kelsh, R.N. and Eisen, J.S. 2000. The zebrafish colourless gene regulates development of non-ectomesenchymal neural crest derivatives. Development 127: 515-525.

Knight, R.D., Nair, S., Nelson, S.S., Afshar, A., Javidan, Y., Geisler, R., Rauch, G.J., and Schilling, T.F. 2003. lockjaw encodes a zebrafish tfap2a required for early neural crest development. Development 130: 5755-5768.

Langenau, D.M., Jette, C., Berghmans, S., Palomero, T., Kanki, J.P., Kutok, J.L., and Look, A.T. 2005. Suppression of apoptosis by bcl-2 overexpression in lymphoid cells of transgenic zebrafish. Blood 105: 3278-3285.

Le Douarin, N. and Kalcheim, C. 1999. The neural crest. Cambridge University Press, New York.

Lister, J.A., Cooper, C., Nguyen, K., Modrell, M., Grant, K., and Raible, D.W. 2006. Zebrafish Foxd3 is required for development of a subset of neural crest derivatives. Dev. Biol. 290: 92-104.

Liu, T.X., Howlett, N.G., Deng, M., Langenau, D.M., Hsu, K., Rhodes, J., Kanki, J.P., D'Andrea, A.D., and Look, A.T. 2003. Knockdown of zebrafish Fancd2 causes developmental abnormalities via p53-dependent apoptosis. Dev. Cell 5: 903-914.

Nakagawa, S. and Takeichi, M. 1998. Neural crest emigration from the neural tube depends on regulated cadherin expression. Development 125: 2963-2971.

Nissen, R.M., Yan, J., Amsterdam, A., Hopkins, N., and Burgess, S.M. 2003. Zebrafish foxi one modulates cellular responses to Fgf signaling required for the integrity of ear and jaw patterning. Development 130: 2543-2554.

Peterson, S.N., Trabalzini, L., Brtva, T.R., Fischer, T., Altschuler, D.L., Martelli, P., Lapetina, E.G., Der, C.J., and White II, G.C. 1996. Identification of a novel RalGDS-related protein as a candidate effector for Ras and Rap1. J. Biol. Chem. 271: 29903-29908.

Robu, M.E., Larson, J.D., Nasevicius, A., Beiraghi, S., Brenner, C., Farber, S.A., and Ekker, S.C. 2007. p53 activation by knockdown technologies. PLoS Genet. 3: e78. doi: 10.1371/journal.pgen.0030078.

Sanchez-Martin, M., Rodriguez-Garcia, A., Perez-Losada, J., Sagrera, A., Read, A.P., and Sanchez-Garcia, I. 2002. SLUG (SNAI2) deletions in patients with Waardenburg disease. Hum. Mol. Genet. 11: 3231-3236.

Shao, H. and Andres, D.A. 2000. A novel RalGEF-like protein, RGL3, as a candidate effector for rit and Ras. J. Biol. Chem. 275: 26914-26924.

Solnica-Krezel, L. 2006. Gastrulation in zebrafish-All just about adhesion? Curr. Opin. Genet. Dev. 16: 433-441.

Stewart, R.A., Arduini, B.L., Berghmans, S., George, R.E., Kanki, J.P., Henion, P.D., and Look, A.T. 2006. Zebrafish foxd3 is selectively required for neural crest specification, migration and survival. Dev. Biol. 292: 174-188.

Su, L., Hattori, M., Moriyama, M., Murata, N., Harazaki, M., Kaibuchi, K., and Minato, N. 2003. AF-6 controls integrin-mediated cell adhesion by regulating Rap1 activation through the specific recruitment of RaplGTP and SPA-1. J. Biol. Chem. 278: 15232-15238.

Trokovic, N., Trokovic, R., Mai, P., and Partanen, J. 2003. Fgfrl regulates patterning of the pharyngeal region. Genes \& Dev. 17: 141-153.

Tsai, I.C., Amack, J.D., Gao, Z.H., Band, V., Yost, H.J., and Virshup, D.M. 2007. A Wnt-CKI $\varepsilon-$ Rap1 pathway regulates gastrulation by modulating SIPA1L1, a Rap GTPase activating protein. Dev. Cell 12: 335-347.

Wieland, I., Jakubiczka, S., Muschke, P., Cohen, M., Thiele, H., Gerlach, K.L., Adams, R.H., and Wieacker, P. 2004. Mutations of the ephrin-B1 gene cause craniofrontonasal syndrome. Am. I. Hum. Genet. 74: 1209-1215.

Zhadanov, A.B., Provance Jr., D.W., Speer, C.A., Coffin, J.D., Goss, D., Blixt, J.A., Reichert, C.M., and Mercer, J.A. 1999. Absence of the tight junctional protein AF-6 disrupts epithelial cell-cell junctions and cell polarity during mouse development. Curr. Biol. 9: 880-888.

Zhang, Z., Rehmann, H., Price, L.S., Riedl, J., and Bos, J.L. 2005. AF6 negatively regulates Rapl-induced cell adhesion. J. Biol. Chem. 280: 33200-33205. 


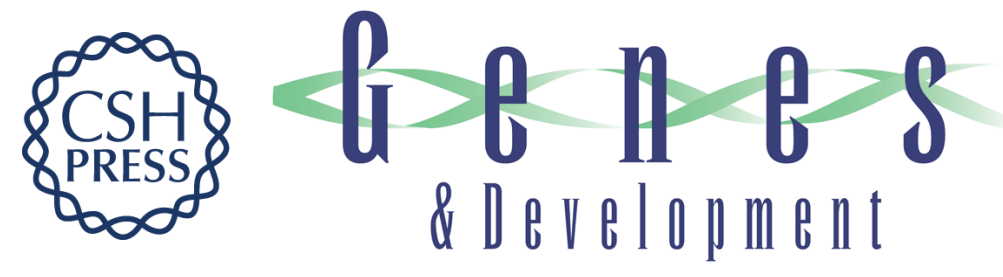

\section{A Rap GTPase interactor, RADIL, mediates migration of neural crest precursors}

Gromoslaw A. Smolen, Benjamin J. Schott, Rodney A. Stewart, et al.

Genes Dev. 2007, 21: originally published online August 17, 2007

Access the most recent version at doi:10.1101/gad.1561507

\section{Supplemental http://genesdev.cshlp.org/content/suppl/2007/08/16/gad.1561507.DC1 Material}

References This article cites 33 articles, 15 of which can be accessed free at: http://genesdev.cshlp.org/content/21/17/2131.full.html\#ref-list-1

\section{License}

Email Alerting

Receive free email alerts when new articles cite this article - sign up in the box at the top Service 